\title{
Changes in Articulation and Phonological Patterns During Early Intervention in Children with Cleft Palate With or Without Cleft Lip
}

\author{
Jennifer Philp ${ }^{* 1}$, Ariel Vovakes ${ }^{1}$, Hope Lancaster ${ }^{1}$, Jennifer Frey ${ }^{2}$, Nancy Scherer ${ }^{1}$
}

Early intervention is often recommended for children with cleft palate, although speech outcomes over the course of intervention have not been extensively documented. The primary objective of this study was to describe the change in use of deletions and substitutions in speech over the course of early intervention for children with cleft palate, with or without cleft lip (CP +/- L). Data from 25 participants were included in this analysis. The participants were part of a randomized control trial comparing Enhanced Milieu Teaching with Phonological Emphasis (EMT+PE) to a business-as-usual (BAU) group. Participants' speech errors were evaluated at four time points using the Profiles of Early Expressive Phonological Skills. The number of deletions and substitutions was assessed at each time point. Further investigation of substitutions was accomplished by comparing cleft-related speech substitutions to developmentally appropriate substitutions. Deletions increased from pre-intervention to mid-point then decreased through the three-month follow-up in both groups. Substitutions increased, decreased, and finally increased again from pre-intervention to the three-month follow-up. Cleft-related substitutions and developmental substitutions did not have the same growth pattern. These results suggest that evaluation of intervention effects must focus on changes in a child's sound system beyond speech accuracy and note changes that mark speech sound progress toward adult-like productions. Additionally, moderate increases in speech errors at the three-month follow-up in the EMT $+\mathrm{PE}$ group indicate the need for continued support following the course of intervention.

\section{INTRODUCTION}

Cleft palate with or without cleft lip $(\mathrm{CP}+/ \mathrm{L})$ is a craniofacial abnormality affecting approximately 1 in 750 live births in the United States each year which negatively impacts speech development (ACPA, 2009). The current understanding of early speech and language development of children with $\mathrm{CP}+/$ - $\mathrm{L}$ indicates that surgical palate repair (typically by 12 months of age) alone is not sufficient to normalize speech development (Jones, Chapman \& Hardin-Jones, 2003). Understanding the factors that impact early speech development is critical to creating effective early speech intervention for these children. One such factor is how speech errors change over time in response to intervention.

\footnotetext{
${ }^{1}$ Department of Speech and Hearing Science, Arizona State University, 975 S. Myrtle Ave, Tempe, AZ 85281

${ }^{2}$ Graduate School of Education and Human Development, George Washington University, 2136 G St NW, Washington D.C. 20052

*To whom correspondence should be addressed: jphilp714@gmail.com
}

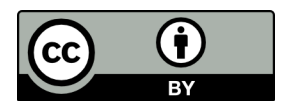

Except where otherwise noted, this work is licensed under https://creativecommons.org/licenses/by/4.0

doi:10.22186/jyi.35.1.20-28
This study investigated speech errors and the role that they play in the participants' speech development. Speech encompasses articulation (i.e., physical production of sound) and phonology (i.e., rules that control the use of sounds in words). Errors can occur at either level. Young children typically display a developmental progression in their learning of the rules of speech sound production that results in adult-like word pronunciation (StoelGammon \& Cooper, 1984). Once children start using their first words at about 12 months of age, they enter a period of highly variable sound production during which they are experimenting with how to produce sounds in words (Stoel-Gammon, 2011). During this process, their speech is characterized by many speech errors such as sound deletions and substitutions (Sosa \& Stoel-Gammon, 2012). Progress from one stage to the next indicates a maturing sound system. One of the early signs of progress towards acquiring a mature sound system is reduction in use of consonant deletions. That is, "mo" becomes "mom" and "ajamas" becomes "pajamas". Simplification of word forms would indicate a less mature phonological system. A second sign of progress is the reduction of consonant substitutions in which one sound replaces another in systematic ways such as "wabbit" becomes "rabbit" or "tat" becomes "cat". Substitutions reflect the child's active generation of speech production rules and these rules progressively approach adult pronunciation patterns. 
In addition to the phonological errors that are expected as a part of typical development, (i.e., cluster reduction, gliding, etc.), children with $\mathrm{CP}+/-\mathrm{L}$ may develop rule-based articulation errors to compensate for their altered anatomy (Pamplona et al., 2000). For children with cleft palate, substitutions and deletions can be considered both articulation and phonological errors. They may be considered articulation errors because children with cleft palate will often replace one sound for another that is easier for them to physically produce. Errors can also be considered phonological because children have developed patterns to replace whole classes of sounds that were made difficult due to their cleft. These patterns persist even after surgical intervention to provide a functional oral mechanism (Chapman, Hardin-Jones, \& Halter, 2003). These "cleft-related substitutions" include the prevalence of substitutions that reflect the inability to close off the nose (i.e., substitution of nasal sounds for other sounds that require high oral air pressure, e.g., "mom" for "pop" or substitution of sounds made in the back of mouth for other sounds, e.g., "cak" for "cat"). Additionally, non-English compensatory sounds are substituted for other sounds, such as a glottal stop in which the vocal cords are used to make a sound in the throat. Essentially, children with $\mathrm{CP}+/-\mathrm{L}$ have missed out on the foundational year on which a typical phonetic inventory would be built. As a result, they use deviant productions to replace whole classes of sounds (Peterson-Falzone, 2017). Because the source of cleft-related errors originates from a physical condition, it is possible that these errors have a different pattern of change over time than errors that are developmentally appropriate for preschool-age children and more phonologically based. It is through inspection of these errors that this study will develop a better understanding of the speech development of children with $\mathrm{CP}+/-\mathrm{L}$ and how it changes during early intervention.

Children with $\mathrm{CP}+/-\mathrm{L}$ receive early speech and language intervention which focuses on expanding vocabulary and simultaneously improving their speech. The goals of early intervention focus on increasing vocabulary, improving speech accuracy, as measured by percent consonants correct, size of consonant inventory, and speech intelligibility to a naive listener (Hardin-Jones, Chapman, Scherer, 2015). One common strategy to improve speech accuracy is to provide the child with a correct pronunciation model when he or she makes speech errors. One evidence based early intervention model that has been shown to reduce speech errors is Enhanced Milieu Teaching with Phonological Emphasis (EMT+PE; Scherer $\&$ Kaiser, 2010). EMT + PE is a naturalistic intervention that aims to provide support for developmental change by focusing on both vocabulary and speech targets. In addition to the targets focused on by EMT such as prompting language in everyday contexts, $\mathrm{EMT}+\mathrm{PE}$ employs the use of speech recasting to promote speech accuracy. EMT $+\mathrm{PE}$ is already documented to improve language and speech accuracy in children with $\mathrm{CP}+/-\mathrm{L}$ (Kaiser, Scherer, Frey, \& Roberts, 2017). This intervention is intended for children once they begin producing early words and focus on training parents to facilitate words in their child's natural environment.

Stoel-Gammon (2011) postulated that as a child's vocabulary develops, his or her sound system matures. The reasoning behind this development is that as more words are acquired, the more a child gets to practice pronunciation of those words. Although naturalistic early speech and language strategies are commonly employed to support early speech and language, to date, no research has examined how speech errors change over time during intervention for children with cleft palate. Therefore, the purpose of this study is to understand how early speech and language intervention changes the use of consonant deletions, developmental substitutions, and cleft-related substitutions to provide the most efficient intervention. Additionally, this study will investigate whether EMT+PE decreases the use of speech errors above and beyond that of a business as usual (BAU) control group. This study will add to the current knowledge regarding the speech development in children with $\mathrm{CP}+/ \mathrm{L}$ as their speech errors change during intervention. In response to this purpose, two research questions will be addressed:

1. Do changes in use of deletions and substitutions differ for children with $\mathrm{CP}+/ \mathrm{L}$ in an $\mathrm{EMT}+\mathrm{PE}$ intervention compared to a business-as-usual control group from pre-intervention to a 3-month follow-up?

2. Does the proportional use of cleft-related and developmental substitutions change over time and is this change the same for both categories?

For the first research question, this study hypothesizes that from pre-intervention to 3-month follow-up, children in the EMT+PE group will decrease their use of deletions to a greater degree than those in the BAU group. As they develop new sounds, children in the EMT+PE group will increase their use of age appropriate developmental substitutions more rapidly than children in the BAU group. With respect to the second research question, this study hypothesizes that cleft-related substitutions will decrease from pre-intervention to 3-month follow-up while developmental substitutions increase and take the place of cleft-related substitutions. This study aims to use these results to aid early speech intervention practices in being as efficient as possible in facilitating typical speech milestones.

\section{METHODS}

The study was approved by Vanderbilt University and East Tennessee State University. This project was an undergraduate project using complete data from a larger study (see Kaiser et al., 2017).

\section{Participants}

Thirty children with $\mathrm{CP}+/-\mathrm{L}$ participated in a longitudinal study of an early speech intervention. Recruitment for this study was based on fliers sent to families identified as having toddlers with cleft palate by cleft palate teams in hospitals, physician's offices, speech and hearing clinics, or through the state early intervention programs and regional health departments. Additionally, fliers were distributed at local preschools, childcare centers, and cleft palate groups as well as shared on the project webpage and Facebook page.

To participate in the larger study, children must: (a) have been 


\begin{tabular}{|c|c|c|}
\hline & $\begin{array}{c}\text { BAU } \\
(n=13)\end{array}$ & $\begin{array}{c}\mathrm{EMT}+\mathrm{PE} \\
(n=12)\end{array}$ \\
\hline $\begin{array}{l}\text { Age at pre-interven- } \\
\text { tion }\end{array}$ & $\begin{array}{l}23.92(6.86) \\
{[15-34]}\end{array}$ & $\begin{array}{l}22.17(6.12) \\
{[13-33]}\end{array}$ \\
\hline Age at palatal repair & $\begin{array}{l}10.42(1.82) \\
{[7-14]}\end{array}$ & $\begin{array}{l}11.92(2.27) \\
{[9-17]}\end{array}$ \\
\hline CDI & $217.17(255.41)$ & $129.75(140)$ \\
\hline Gender breakdown & $\begin{array}{l}\text { Female: } 5 \\
\text { Male: } 8\end{array}$ & $\begin{array}{l}\text { Female: } 5 \\
\text { Male: } 7\end{array}$ \\
\hline Cleft type & $\begin{array}{l}\text { Cleft palate only: } 4 \\
\text { Unilateral cleft lip } \\
\text { and palate: } 7 \\
\text { Bilateral cleft lip and } \\
\text { palate: } 2\end{array}$ & $\begin{array}{l}\text { Cleft palate only: } 3 \\
\text { Unilateral cleft lip } \\
\text { and palate: } 6 \\
\text { Bilateral cleft lip and } \\
\text { palate: } 3\end{array}$ \\
\hline Mother's Education & $\begin{array}{l}\text { Some college or two- } \\
\text { year degree: } 4 \\
\text { Four-year degree or } \\
\text { more: } 8 \\
\text { Unreported: } 1\end{array}$ & $\begin{array}{l}\text { High School or } \\
\text { GED: } 3 \\
\text { Some college or two- } \\
\text { year degree: } 3 \\
\text { Four-year degree or } \\
\text { more: } 6\end{array}$ \\
\hline
\end{tabular}

Table 1. Descriptive information by treatment groups. Mean age in months (Standard deviation) [age range in months] The MacArthur-Bates Communicative Development Inventory (CDI) was administered as a parent-reported measure of expressive vocabulary before intervention.

between thirteen and thirty six months of age; (b) have a composite score of 80 or above on the Bayley Scales of Infant and Toddler Development-III (Bayley-III; Bayley, 2006); (c) have been able to produce at least five different words per parent report on the MacArthur Bates Communicative Development Inventory (MCDI; Fenson et al., 2007); and (d) have demonstrated at least one type of articulatory error during screening of the Profiles of Early Expressive Phonological Skills (PEEPS; Stoel-Gammon \& Williams, 2013) and/or during the language sample. These errors could include: (a) a compensatory error replacing at least one phoneme; (b) a consonant inventory of less than five stop or nasal consonants in all positions; and/or (c) errors on at least two stop or nasal consonants.

Children were excluded from the larger study if they (a) had a sensorineural hearing loss or a sound-field hearing threshold of over $30 \mathrm{~dB} \mathrm{HL}$, as reported by an audiologist or confirmed by the medical record; (b) were multilingual or non-English speaking based on parent report; (c) had a syndrome diagnosis from a geneticist; and/or (d) had more than three dysmorphic features in addition to the cleft palate. To be included in the larger study it was not necessary to have a diagnosis of a speech or language impairment. At pre-intervention all participants ranged from thirteen to thirty-four months of age and all participants had their palates surgically repaired between seven and seventeen months of age.

\section{Intervention}

Children were randomly assigned to either the Enhanced Milieu Teaching with Phonological Emphasis intervention group or the business-as-usual control group.

Toddlers included in the intervention group received $\mathrm{EMT}+\mathrm{PE}$. The intervention group received 48 30-minute sessions twice weekly over the course of six months. Each intervention session was individualized and led by a speech language pathologist trained in EMT+PE strategies. EMT+PE is a naturalistic intervention and an adaptation of Enhanced Milieu Teaching (Kaiser et al., 1998). During an intervention session, toys were selected based on child-specific sound targets and arranged to elicit child-initiated interactions (environmental arrangement). Clinicians would model speech and engage the child while connecting speech productions to the child's play and specific interests (responsive interaction). Feedback given to the child was aimed at providing access to child requested objects and advanced pronunciations given the child's targeted skills (milieu teaching). The addition of phonological emphasis (EMT+PE) introduced the component of speech recasting to focus on speech accuracy skills. Recasting provides a phonologically accurate production in response to an imprecise production made by the child.

Toddlers included in the BAU group did not receive EMT $+\mathrm{PE}$ intervention. Rather, individuals in this group were free to participate in other intervention treatments and continue to follow up with their cleft palate teams.

\section{Measures}

Participants' speech was assessed using the Profiles of Early Expressive Phonological Skills (PEEPS, Stoel-Gammon \& Williams, 2013) assessment at four time points (pre-intervention, midpoint, post-intervention, three-month follow-up). Assessments took place over the course of nine months. The PEEPS assessment contains 40 age appropriate single words designed to represent the full array of possible place and manner productions as well as a variety of word shapes. The assessment employs the use of toys to engage the child in age-appropriate play. Toys are used in response to child-initiated interactions with the clinician. At pre-intervention, 12 participants received a preliminary PEEPS assessment consisting of 12 words due to limited word use. The PEEPS assessment was scored by a speech language pathologist (SLP) not involved in data collection but familiar with the transcription of cleft palate speech. Intra and inter reliability was performed on the phonetic transcriptions. Interobserver agreement was calculated for each coded entry. If interobserver agreement fell below $85 \%$ for a category at any time point, the two coders met to discuss the coding discrepancy and came to a conclusion.

All PEEPS transcriptions were imported into the PHON database (Byun \& Rose, 2016). PHON is a computer software used to transcribe and analyze phonological data. Queries on percent consonants correct (PCC), phonological mean length of utterance (pMLU) and consonant inventories were performed. PHON data was then exported to an Excel workbook. Reliability was performed on the Excel data entry for all participants who had complete pre-intervention and post-intervention PEEPS information by an SLP not involved in the original data entry. The data entry for this sample had 100\% agreement between the two researchers. 


\begin{tabular}{|c|c|c|c|c|c|c|c|c|}
\hline & \multicolumn{2}{|c|}{ Pre-Intervention } & \multicolumn{2}{|c|}{ Midpoint } & \multicolumn{2}{|c|}{ Post-Intervention } & \multicolumn{2}{|c|}{ Follow-Up } \\
\hline & BAU & $\mathrm{EMT}+\mathrm{PE}$ & BAU & $\mathrm{EMT}+\mathrm{PE}$ & BAU & $\mathrm{EMT}+\mathrm{PE}$ & BAU & $\mathrm{EMT}+\mathrm{PE}$ \\
\hline PCC & $\begin{array}{c}47.24 \\
(13.96)\end{array}$ & $\begin{array}{c}32.41 \\
(12.87)\end{array}$ & $\begin{array}{c}55.06 \\
(20.48)\end{array}$ & $\begin{array}{c}41.71 \\
(17.97)\end{array}$ & $\begin{array}{c}63.21 \\
(20.51)\end{array}$ & $\begin{array}{c}56.54 \\
(22.96)\end{array}$ & $\begin{array}{c}69.26 \\
(17.36)\end{array}$ & $\begin{array}{c}61.37 \\
(22.24)\end{array}$ \\
\hline pMLU & $\begin{array}{c}3.70 \\
(0.68)\end{array}$ & $\begin{array}{c}3.07 \\
(0.58)\end{array}$ & $\begin{array}{c}3.90 \\
(0.88)\end{array}$ & $\begin{array}{c}3.50 \\
(0.84)\end{array}$ & $\begin{array}{c}4.41 \\
(0.73)\end{array}$ & $\begin{array}{c}4.09 \\
(0.82)\end{array}$ & $\begin{array}{c}4.61 \\
(0.55)\end{array}$ & $\begin{array}{c}4.29 \\
(0.79)\end{array}$ \\
\hline Substitutions & $\begin{array}{c}12.77 \\
(11.61)\end{array}$ & $\begin{array}{c}12.25 \\
(11.67)\end{array}$ & $\begin{array}{c}16.77 \\
(11.60)\end{array}$ & $\begin{array}{l}16.83 \\
(7.25)\end{array}$ & $\begin{array}{l}16.83 \\
(6.74)\end{array}$ & $\begin{array}{c}16 \\
(8.55)\end{array}$ & $\begin{array}{l}15.31 \\
(8.42)\end{array}$ & $\begin{array}{c}17.67 \\
(11.85)\end{array}$ \\
\hline Deletions & $\begin{array}{c}5.54 \\
(3.84)\end{array}$ & $\begin{array}{c}11.33 \\
(11.79)\end{array}$ & $\begin{array}{c}9.38 \\
(7.94)\end{array}$ & $\begin{array}{l}16.50 \\
(9.04)\end{array}$ & $\begin{array}{c}8.31 \\
(9.72)\end{array}$ & $\begin{array}{l}14.08 \\
(9.70)\end{array}$ & $\begin{array}{c}5.54 \\
(4.74)\end{array}$ & $\begin{array}{l}10.75 \\
(9.53)\end{array}$ \\
\hline
\end{tabular}

Table 2. Longitudinal Speech Characteristics by Treatment Groups. Mean (Standard Deviation). Percent Consonants Correct (PCC) and Phonological Mean Length of Utterance (pMLU) calculated using PHON.

Participant's speech errors were coded using a set of 17 error categories. These categories were grouped into the following categories: substitutions (e.g., developmental substitutions and cleft-related substitutions), and consonant deletions (see Table 4. for error category definitions). Developmental substitutions include errors that are expected while acquiring speech in typically developing toddlers (e.g., fronting, stopping, gliding). Alternatively, cleft-related substitutions are errors that are a result of the altered anatomy that a child with $\mathrm{CP}+/-\mathrm{L}$ would have. This altered anatomy leads to cleft-related substitutions, which include nasalization, backing and compensatory errors such as glottal stops, pharyngeal fricatives, velar fricatives, and posterior nasal fricatives. Consonant deletions would include the omission of any consonant where there is no attempt to replace it with another phoneme.

\section{Analysis}

Data were compiled and stored in Excel. Most of the data processing, analysis, and visualizations was completed in $\mathrm{R}$ (Version 3.3.2; R Core Team, 2016). The following packages were used: readxl (Wickham, 2016), psych (REvelle, 2016), dplyr (Wickham \& Francois, 2016), ggplot2 (Wickham, 2009), tidyr (Wickham, 2017), knitr (Xie, 2016), reshape2 (Wickham, 2007), and nlme (Pinheriro, Bates, DebRoy, Sarkar, \& R Core Team, 2016). The code used for this project is publicly shared and available online (Lancaster, 2017).

This study tested for group differences at pre-intervention using Welch's two sample t-test for independent groups with unequal variances. To address research question one, growth curve modeling was used to determine the best fitting model for change over time using maximum likelihood. This study first examined fixed effects of time, time-squared (quadratic term), and time-cubed (cubic term). Models were compared using chi-square tests to determine the best fitting model. After determining the best fitting slope model, the intervention group was entered as a fixed effect (conditional model) and compared the conditional model to the previous model to determine if there was a main effect. If the addition of the intervention group improved model fit, an interaction term was added and compared to the condition model. Intercepts were allowed to vary by participant. P-values were obtained using likelihood tests comparing the full model against the model without the effect in question.

For research question two, the proportion of cleft-related substitutions to developmental substitutions out of total substitutions over time were visually compared. Chi-square tests of independence were used to examine the change in use of cleft-related and developmental substitutions; specifically, the relationship between intervention group and time and the relationship between intervention group and type of substitution was examined. Four 2 by 2 contingency tests were done to compare proportions of compensatory and developmental errors between EMT+PE and BAU at each time. Two 2 by 4 contingency tests compared EMT $+\mathrm{PE}$ to BAU across time within each error type (pre-intervention to follow-up). Four 2 by 2 post-hoc comparisons were done to examine change from pre-intervention to post-intervention and from post-intervention to follow up between groups within error type. Chi-square tests were used instead of analysis of variance because the data was expected to violate the assumption of equal variances. Scores were converted to proportions to adjust for low counts. Chi-square tests were completed using Excel.

\section{RESULTS}

At pre-intervention, children in the EMT+PE group had smaller parent reported vocabulary as seen in Table 1, but this difference was not significant $(t(17.06)=1.04, p=0.313)$. The means and standard deviations for speech accuracy and speech error measures over the course of intervention are reported in Table 2. The EMT + PE group had a significantly lower PCC at pre-intervention $(t(21.26)=3.217, p=0.04)$, which means that the EMT $+\mathrm{PE}$ group had less accurate consonant productions at pre-intervention than the BAU group. The distribution for deletion and substitution errors indicated that errors were not normally distributed. However, non-normal distribution of child speech errors is common for young children. Some of the participants had zero errors at pre-intervention because they produced no consonant sounds. It was therefore important to assess whether the groups differed in their use of speech errors of interest for this study at pre-intervention. The EMT $+\mathrm{PE}$ and BAU groups did not significantly differ on number of deletions $(t(13.15)=-1.63, p=0.128)$ or substitu- 


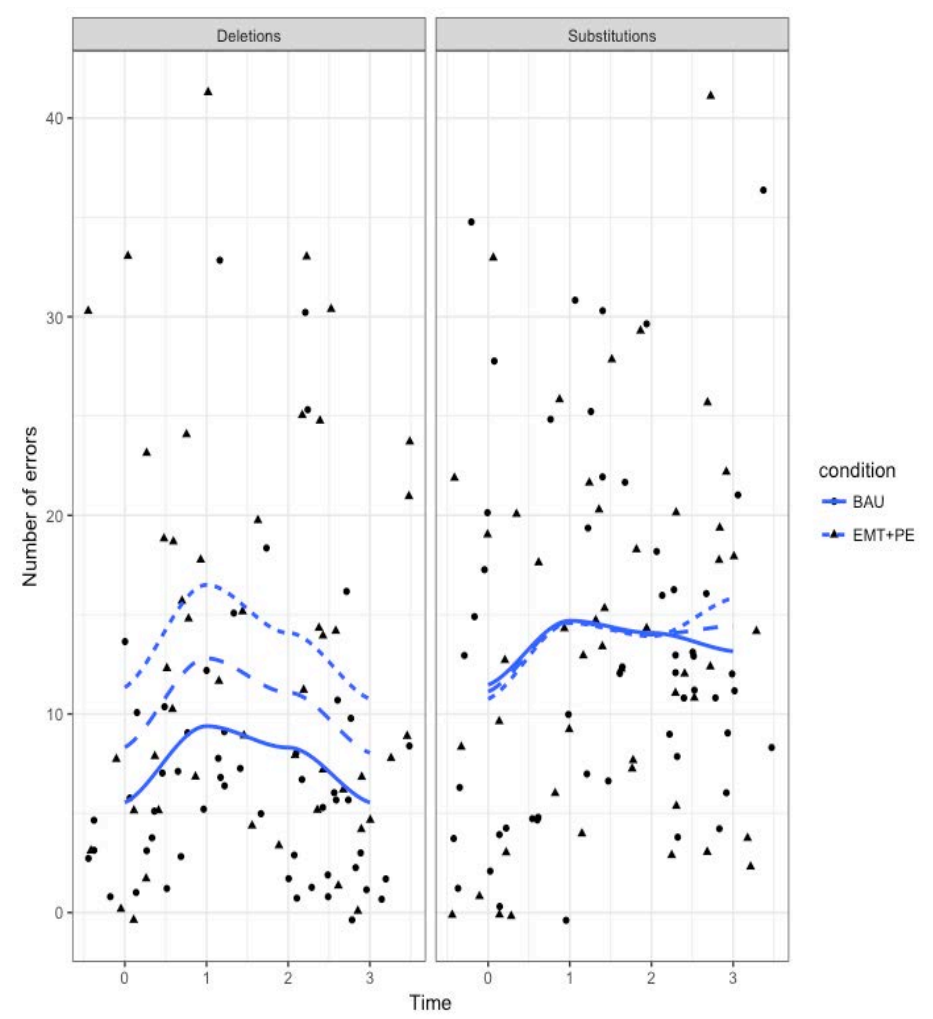

Figure 1. Total number of errors produced for each subject for deletions (left panel) and substitutions (right panel) across time by intervention group. Fitted lines are also plotted. The blue medium dash line is the fitted line for the whole sample. The BAU group is the solid line, whereas the EMT $+\mathrm{PE}$ group is the dotted line. The circles represent scores with the BAU group, whereas the EMT $+\mathrm{PE}$ scores are represented as triangles.

tions $(t(22.95)=0.162, p=0.873)$ at pre-intervention; although, the EMT+PE groups did have more deletions than the BAU group over the entire course of intervention. This lack of statistical difference implies that the proposed analyses can be carried out as planned, but these pre-intervention differences will need to be considered when interpreting the results. Total error use, deletions and substitutions, by intervention group and time with best fit lines are plotted in Figure 1.

\section{Changes in Error Use Over Time and Effect of Intervention} Group

\section{Deletions}

Model statistics for deletions over time are displayed in Table 3. Adding the quadratic term (Model 2) improved the prediction of deletions $\left(\chi^{2}(1)=7.01, p=0.008\right)$ compared to the linear model (Model 1); but, adding the cubic term (Model 3 ) did not improve on Model $2\left(\chi^{2}(1)=0.62, p=0.431\right)$. These comparisons indicated that the best fitting model for the data was the quadratic model (Model 2). The quadratic term was negative, which means that by follow-up all children had decreased their use of deletions compared to pre-intervention by 1 to 2 errors (on average).
To explore the effect of intervention group, intervention group was added to the model There was a significant model improvement for adding intervention group (Model 4) $\left(\chi^{2}(1)=6.08, p=\right.$ $0.014)$ compared to Model 2 (quadratic). Adding an interaction term between intervention group and the quadratic slope term did not improve model fit (Model 5) $\left(\chi^{2}(1)=0.11, p=0.737\right)$ compared to Model 4. The final model for deletions was Model 4 which contained linear and quadratic slope terms and the conditional variable of intervention group. There was a main effect of intervention group in Model 4 indicating that the intercept significantly differed between the groups. Specifically, children in the EMT $+\mathrm{PE}$ had significantly more deletions, $5.97(+/-2.32)$, than children in the BAU group at pre-intervention. However, there was no difference between the two groups for slope, suggesting that although the EMT+PE group began intervention with more deletions, all children had the same trajectory for decreasing their deletions over time.

Substitutions

The linear term for Model 6 was not significant $(t(74)=1.22, p$ $=0.2233)$ and the slope term was close to zero (0.932); therefore, the model could be not interpreted. Visual analysis of fitted lines (Fig. 1) indicated that substitutions had a cubic change with no differences between intervention and BAU groups for intercept or slope of line. However, modeling a cubic relationship yielded no significant slope terms (linear: $t(72)=1.13, p=0.2636$; quadratic: $t(72)=-0.82, p=0.4174$; cubic: $t(72)=0.69, p=0.4927)$. These results indicated that there was no ideal growth curve model for all substitutions.

Differences in Cleft-Related and Developmental Substitutions Figure 2 provides a graph of total use of cleft-related and developmentally appropriate substitutions by intervention group and time with best fitting lines. Although total substitutions were similar in the two groups (Fig. 1), the use of cleft-related and developmental substitutions (Fig. 2) appeared to differ over time. Visual inspection of Figure 2 indicated that cleft-related substitutions and developmentally appropriate substitutions had different best fitting lines. Cleft-related errors had a negative trend over time, although the EMT+PE group increased errors between post-intervention and follow-up without intervention. In contrast, developmentally appropriate substitutions rapidly increased from pre-intervention to mid-point for both groups. The EMT $+\mathrm{PE}$ group continued to increase in their use of developmentally appropriate substitutions, whereas the BAU group plateaued after mid-point. These two different patterns of change over time may explain why neither of the growth curve models fit.

Furthermore, visual analysis suggests that there were differences in the type of substitutions. The EMT+PE group had significantly higher proportions of cleft-related substitution mid-point $\left(\chi^{2}(1)=12.55, p=0.0004\right)$ and follow-up $\left(\chi^{2}(1)=6.04, p=0.014\right)$ than the BAU group, but groups did not differ at pre-intervention $\left(\chi^{2}(1)=2.99, p=0.084\right)$ or post-intervention $(2(1)=0.0437, p=$ $0.834)$.

Figure 3 displays the change in proportion out of total errors 


\begin{tabular}{lllllll}
\hline Predictor & Ceof (B) & SE (B) & $z$ & $p$ & $\begin{array}{r}95 \% \mathrm{CI} \\
\text { (lower) }\end{array}$ & $\begin{array}{l}95 \% \mathrm{CI} \\
\text { (upper) }\end{array}$
\end{tabular}

\begin{tabular}{ccccccc}
\hline \multicolumn{7}{c}{ Model 1 } \\
\hline Intercept & 10.44 & 1.63 & 6.41 & 0.0000 & 7.23 & 13.66 \\
Linear slope & -0.256 & 0.66 & -0.39 & 0.6977 & -1.55 & 1.04 \\
\hline
\end{tabular}

Model 2

\begin{tabular}{ccccccc}
\hline Intercept & 8.56 & 1.76 & 4.87 & 0.0000 & 5.11 & 12.01 \\
\hline Linear slope & 5.38 & 2.20 & 2.44 & 0.0170 & 0.06 & 9.71 \\
Quadratic slope & -1.88 & 0.70 & -2.67 & 0.0093 & -3.26 & -0.49 \\
\hline \multicolumn{7}{c}{ Model 3 } \\
\hline Intercept & 8.32 & 1.79 & 4.64 & 0.0000 & 4.82 & 11.82 \\
\hline Linear slope & 9.20 & 5.41 & 1.70 & -0.930 & -1.36 & 19.77 \\
Quadratic slope & -5.54 & 4.78 & 1.16 & 0.2503 & -14.88 & 3.79 \\
\hline Cubic slope & 0.81 & 1.05 & 0.77 & 0.4414 & -1.24 & 2.87 \\
\hline & & Model 4 & & & \\
\hline Intercept & 5.69 & 1.99 & 2.85 & 0.0057 & 1.79 & 9.59 \\
\hline Linear slope & 5.38 & 2.22 & 2.43 & 0.0176 & 1.06 & 9.71 \\
\hline Quadratic slope & -1.88 & 0.71 & -2.66 & 0.0097 & -3.26 & -0.49 \\
\hline $\begin{array}{c}\text { Intervention } \\
\text { (EMT+PE) }\end{array}$ & 5.97 & 2.32 & 2.57 & 0.0171 & 1.26 & 10.68 \\
\hline
\end{tabular}

Model 5

\begin{tabular}{ccccccc}
\hline Intercept & 5.47 & 2.12 & 2.58 & 0.0119 & 1.35 & 9.59 \\
\hline Linear slope & 5.38 & 2.23 & 2.42 & 0.0181 & 1.06 & 9.71 \\
\hline Quadratic slope & -1.82 & 0.74 & -2.46 & 0.0162 & -3.25 & 11.95 \\
\hline $\begin{array}{c}\text { Intervention } \\
\text { (EMT+PE) }\end{array}$ & 6.44 & 2.74 & 2.35 & 0.0275 & 0.93 & 11.95 \\
\hline $\begin{array}{c}\text { Interaction } \\
\left(\begin{array}{c}\text { EMT+PE * } \\
\text { time }\end{array}\right.\end{array}$ & -0.13 & 0.41 & -0.33 & 0.7444 & -0.92 & 0.66 \\
\hline
\end{tabular}

Model summary statistics

\begin{tabular}{ccccc}
\hline Model & AIC & BIC & ICC & logLik \\
\hline Model 1 & 716.93 & 727.35 & 0.35 & -354.47 \\
\hline Model 2 & 711.92 & 724.95 & 0.38 & -350.96 \\
Model 3 & 713.29 & 728.93 & 0.38 & -350.65 \\
Model 4 & 707.84 & 723.47 & 0.29 & -347.92 \\
\hline Model 5 & 709.73 & 727.96 & 0.29 & -347.86 \\
\hline
\end{tabular}

Table 3. Growth Curve Model Information and Model Comparisons for Deletions Across Time. Result summary for fixed effects for deletions over time: coefficient estimates (B), standard errors SE (B), associated t-score, significance level, and 95\% confidence interval for each predictor for all models and model summary statistics. by time point and intervention group. The change across all four time points for cleft-related and developmental substitutions were examined. The observed proportions for cleft-related substitutions $\left(\chi^{2}(3)=5.51, p=0.138\right)$ and developmental substitutions $\left(\chi^{2}(3)=3.15, p=0.368\right)$ did not differ over time between the groups. Because the $2 \mathrm{X} 4$ contingency tests may have missed subtle changes at key times points, post-hoc analysis compared the proportions between pre- and post-intervention and post-intervention and follow-up. The groups did not differ over time for cleft-related (pre-post: $\chi^{2}(1)=0.54$, df $=1, p=0.459$; postfollow up: $\chi^{2}(1)=2.76, p=0.096$ ) or developmental substitutions (pre-post: $\chi^{2}(1)=1.41, p=0.236$; post-follow up: $\left.\chi^{2}(1)=0.96, p=0.328\right)$. Although gains were not sustained from post-intervention to follow-up in the EMT+PE group for cleft-related errors, the EMT + PE group had a higher rate of cleft-related errors at pre-intervention and did not differ from the BAU group at post-intervention. Detailed inspection of the types of cleft-related errors showed that children in the EMT+PE group increased their proportional use of compensatory substitutions from post-intervention $(\mathrm{M}=10.22, \mathrm{SD}=13.86)$ to follow-up $(\mathrm{M}=15.88, \mathrm{SD}=$ 19.12) without intervention.

\section{DISCUSSION}

The purpose of this study was to investigate how speech errors, specifically consonant deletions and substitutions, changed the course of early speech-language intervention in young children with $\mathrm{CP}+/ \mathrm{L}$ who are still acquiring a complete sound system. It was found that the number of consonant deletions and cleft-related substitutions decreased during treatment as the use of developmentally appropriate substitutions increased. Essentially, consonant substitutions replaced deletions. Cleft-related substitutions declined in the intervention group but increased again following intervention suggesting that the new sounds were not yet integrated into conversational use. Below the results are interpreted as they relate to the research questions and hypotheses.

\section{Change in Consonant Deletions and Substitutions}

This study hypothesized that during the course of intervention children in the EMT+PE group would decrease their use of deletions to a greater degree than those in the BAU group. All children increased their use of consonant deletions from pre-intervention to mid-point and then decreased from the mid-point of intervention to the 3-month followup (Fig. 1 and Table 2). The decrease in deletions paired with a steady increase in speech accuracy, as measured by percent consonants correct, throughout the study resulted in a more mature sound system. However, this study was not able to identify a best fitting model for a change in substitutions, as visual inspection of the data revealed that the two types of substitutions, developmental and cleft-related, had 


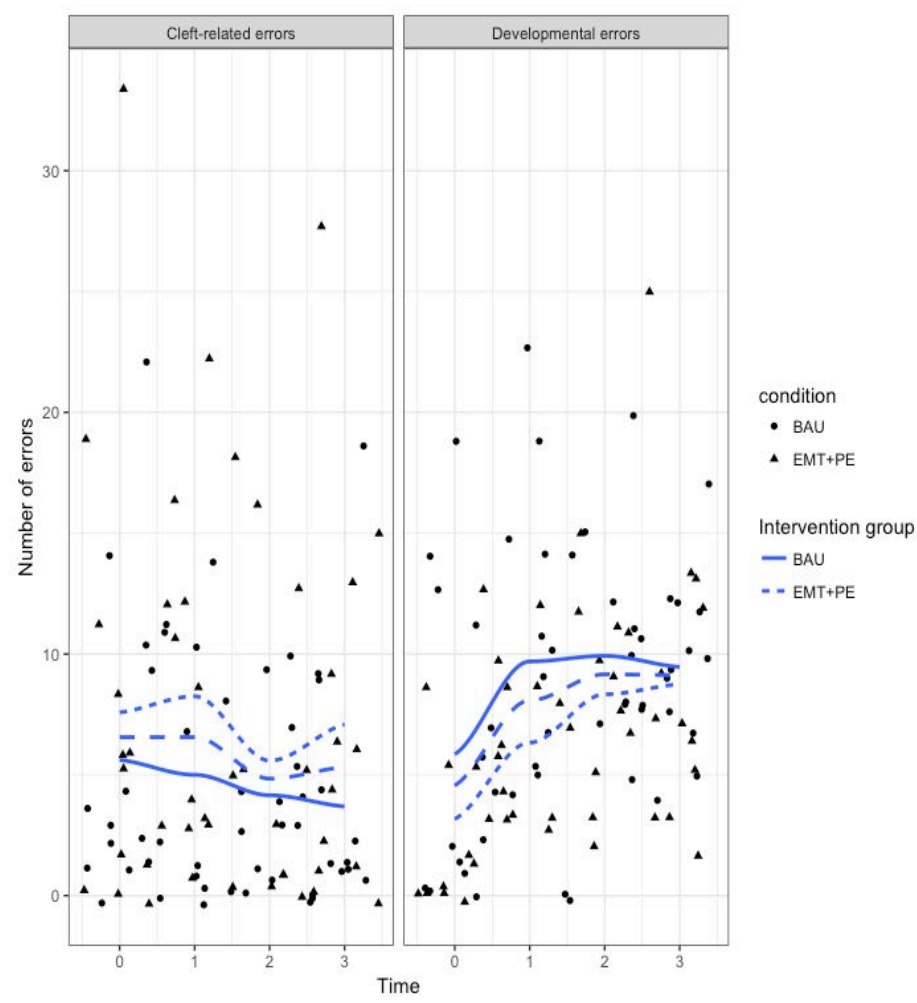

Figure 2. Total number of errors and fitted lines for cleft-related (left panel) and developmental (right panel) substitutions across time by intervention group. The blue medium dash line is the fitted line for the whole sample. The BAU group is the solid line, whereas the EMT+PE group is the dotted line. The circles represent scores with the BAU group, whereas the EMT + PE scores are represented as triangles.

different growth trajectories. All children increased their use of developmental substitutions during intervention, while decreasing their use of cleft-related substitutions. Combined with the change in deletions over time, these results indicate that children with $\mathrm{CP}+/$-L initially omitted consonants or replaced consonants with a cleft-related error, but early intervention helped these children replace their errors with more developmentally appropriate errors.

In this study, the observed change in the participants' speech is indicative of a maturing sound system. Specifically, the decrease in deletions and cleft-related substitutions and increase in use of developmentally expected substitutions shows that the children were developing more stable and rule-based word productions. Additional work with these data indicated that participants' sound systems matured in relation to their vocabulary development (see Kaiser et al., 2017; Scherer, Kaiser, Frey, Roberts, \& Lancaster, under review). Stoel-Gammon (2011) postulated that as vocabularies increase, children have more opportunities to practice sound production, which in turn, improves expressive phonology. In the case of associated current and previous studies, toddlers with $\mathrm{CP}+/-\mathrm{L}$ demonstrated positive development in their sound system by increasing their lexicon and use of developmentally expected

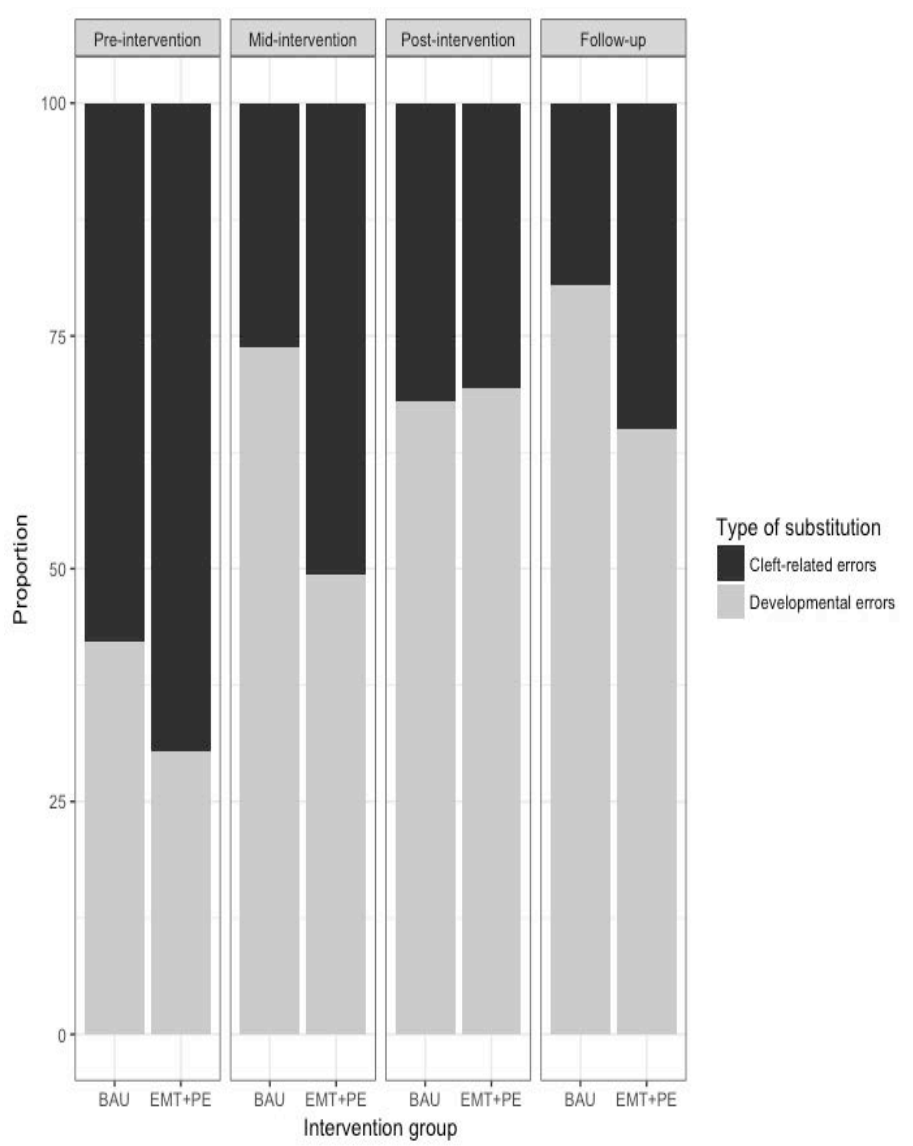

Figure 3. Proportion of cleft-related (black) and development (gray) substitutions out of total substitutions by across time by group.

substitutions and by decreasing their use cleft-related substitutions (Kaiser et al., 2017; Scherer, et al., under review).

Additionally, this study hypothesized that children in the EMT+PE group would normalize their speech production more rapidly than children in the BAU group. There was significant main effect of intervention for consonant deletions. However, the two groups had similar rates of change during the course of intervention. Even though the two groups were not significantly different in their use of substitutions at the onset of the study, the EMT+PE group had a larger proportion of cleft related substitutions which are often more resistant to change in therapy (Peterson-Falzone, 2017). Results from Scherer et al. (under review) suggest that children in the EMT $+\mathrm{PE}$ group who used language more frequently had greater gains in speech production than children who talked less suggesting that individual child characteristics had an impact on their response to intervention.

Changes in Cleft-Related Substitutions Compared to Developmental Substitutions

This study hypothesized that cleft-related substitutions would decrease from pre-intervention to 3-month follow-up while developmental substitutions increased. Substantial support for this 
hypothesis was found. The elimination of reliance on cleft-related speech errors in favor of developmental errors is an important milestone for children with $\mathrm{CP}+/ \mathrm{L}$. For this study, nasal substitutions, backing, and compensatory errors were classified as cleftrelated substitutions. Within the model of decreasing cleft-related errors, inspection of the three time points is important (see Figure 3 ). In the BAU group the most prevalent cleft-related substitution remains backing from pre- to post-intervention where it shifts to a tie between backing and compensatory errors at follow-up. A1ternatively, in the EMT+PE group the most prevalent cleft-related substitutions are compensatory errors at all time points with the exception of post-intervention at which point the most frequent cleft-related substitution becomes backing. Differing proportions, as made within the cleft-related category, illustrate the following point: although changes in both substitution categories are indicative of developmental progress, they account for two fundamentally discrete quantities. Filling in developmental substitutions marks phonological maturation for all children. Decreasing the use of cleft-related substitutions is an added step that toddlers with $\mathrm{CP}+/-\mathrm{L}$ must take to move closer to their typically developing peers. The two categories are not equivalent and therefore do not fit together statistically, which was further supported by the finding that neither of the growth curve models fit the data when substitutions were treated as a single group. Even though the two groups were not significantly different in their use of substitutions at the onset of the study, the EMT+PE group had a larger proportion of cleft-related substitutions which are often more resistant to change in therapy (Peterson-Falzone, 2017). Differing abilities between groups would have impacted individualized intervention targets. Ultimately, intervention targets for both groups were similar, but more deletions in the EMT+PE group would first prompt intervention that focusses on filling in those missing sounds.

\section{Clinical Implications}

This study has two main clinical implications. First, evaluation of intervention effects for children under three years of age should monitor speech change in terms of speech error types as well as standard measures of speech accuracy. Both measurements provide an indication of developmental progress but examining error types may serve as a tool and be the clinician's earliest indication of speech change. Alternatively, speech accuracy provides a measurement of how close the child's speech is to the adult model. Secondly, parents need to be trained to support child speech development by integrating new sounds in conversational use so that speech skills are maintained following the conclusion of intervention. In this study, children in the EMT+PE group showed reduction of compensatory errors following intervention, but these gains were not maintained in follow up.

\section{Limitations and Future Directions}

Methodological limitations must be considered in the interpretation of these results and clinical implications. One limitation of this study is the small sample size. Additionally, despite random sampling, the EMT+PE group started intervention slightly younger and behind the BAU group on both language and speech mea- sures. Despite best efforts to randomize participants and create equivalent groups, children in the EMT+PE group had less accurate speech at pre-intervention as seen by their significantly lower PCC scores. Without equivalent starting points, intervention targets may have differed, possibly impacting results. Initial inspections indicated that the groups did not differ in use of consonant deletions or substitutions, however, the EMT+PE group did have more deletions. Although the t-tests of pre-intervention were not significant, intervention group was significant in the growth curve model for consonant deletions indicated that the groups did significantly differ in their use of consonant deletions at pre-intervention. These pre-intervention differences could be one reason that the hypothesized effects for EMT+PE were not found.

Future research can address the limitations of the present study by increasing the sample size and modifying inclusion criteria by having a minimum number of required words and sounds in a child's inventory, which may lead to fewer discrepancies between treatment groups throughout intervention. The partner study mentioned above found that in EMT $+\mathrm{PE}$ intervention, children with a word frequency of approximately seven words per minute (WPM) are candidates for EMT+PE (Vovakes, Philp, Lancaster, Lien, \& Scherer, 2017). The participants who produced approximately seven WPM at pre-intervention showed the greatest increase in speech accuracy at post-intervention, therefore, future research may want to consider rate of production as additional inclusionary criteria for participants. This study is unable to discuss whether the above results differ from what is developmentally expected in a child without $\mathrm{CP}+/ \mathrm{L}$, as it is beyond the scope of this project. Assessing whether these results go above and beyond developmental expectations could be determined by analyzing the change in speech errors for children in the first word stage or children with cleft palate who have not received early speech and language intervention.

\section{CONCLUSION}

Young children with $\mathrm{CP}+/$-L present a complex picture of anatomical and phonological learning challenges. This study has examined both developmental and cleft-related speech errors during early speech development. The reduction in cleft related errors, particularly compensatory errors, was a significant finding. Traditionally these error types are thought to reflect velopharyngeal dysfunction, which is anatomically based and requires surgical or prosthetic intervention to change the anatomy. However, for young children who are still acquiring speech, these errors may decline following palate surgery commensurate with typical phonological development and not reflect velopharyngeal status at this early age.

Children enrolled in early speech and language intervention make desirable changes in their speech error use as evidenced by increased speech accuracy, decreased use of consonant deletions and cleft-related substitutions, and increased use of developmental substitutions.

\section{ACKNOWLEDGMENTS}


This research was conducted at East Tennessee State University and Vanderbilt University and was supported by the National Institute of Deafness and Other Communication Disorders 1R21DCOO9654. We want to thank the families who participated in this study for their dedication. We also want to acknowledge the efforts of the research team members at Vanderbilt University (Dr. Ann Kaiser), George Washington University, East Tennessee State University, and Arizona State University (Kari Lien). Thank you to Ariel Vovakes, Dr. Hope Lancaster, Dr. Jennifer Frey, and Dr. Nancy Scherer for their guidance and mentorship to the first author. Lastly, thank you to Dr. Yvan Rose for his support as we learned how to use PHON.

\section{REFERENCES}

Bayley, N. (2006). Bayley Scales of Infant and Toddler Development--Third Edition, Screening Test. 17, 2006, Vol.17.

Byun, T., \& Rose, Y. (2016). Analyzing Clinical Phonological Data Using Phon. $37(02), 085-105$.

Chapman, K. L., Hardin-Jones, M., \& Halter, K. A. (2003). The relationship between early speech and later speech and language performance for children with cleft lip and palate. Clinical Linguistics \& Phonetics, 17(3), 173-197. https://doi.org/10.1080/0269920021000047864

Fenson, Larry. (2007). MacArthur-Bates Communicative Development Inventories, Second Edition. 19, 2007, Vol.19.

Girolametto, L., Weitzman, E., \& Clements-Baartman, J. (1998). Vocabulary inter vention for children with Down syndrome: Parent training using focused stimulation. Infant-Toddler Intervention: A Transdisciplinary Journal, 8(2), 109-125.

Hardin-Jones, Chapman, and Scherer, (2015). Children with Cleft Lip and Palate: A Parents' Guide to Early Speech-Language Development \& Treatment. Bethesda, MD: Woodbine House, Inc.

Hardin-Jones, M., Chapman, K., \& Schulte, J. (2003). The impact of cleft type on early vocal development in babies with cleft palate. The Cleft Palate-cranio facial Journal: Official Publication of the American Cleft Palate-Craniofacial Association, 40(5), 453-9.

Kaiser, A. P., Hancock, T. B., \& Hester, P. P. (1998). Parents as co-interventionists: Research on applications of naturalistic language teaching procedures. Infants and Young Children, 10(4), 46-55.

Kaiser, A., Scherer, N., Frey, J., \& Roberts, M. Y. (2017). The effects of Enhanced Milieu Teaching with Phonological Emphasis on the speech and language skills of young children with cleft palate: A pilot study. American Journal of Speech-Language Pathology, Advanced online publication. doi:10.1044/2016_AJSLP-16-0008.

Lancaster, H. (2017). Changes in Articulation and Phonological Patterns During Early Intervention in Children with Cleft Palate with or without Cleft Lip. ResearchGate, DOI: 10.13140/RG.2.2.21906.35523.

Pamplona, M. C., Ysunza, A., Gonzalez, M., Ramirez, E., \& Patino, C. (2000). Linguistic development in cleft palate patients with and without compensatory articulation disorder. International Journal of Pediatric Otorhinolaryngology, 54(2-3), 81-91. https://doi.org/10.1016/S0165-5876(00)00332-3

Parameters for the Evaluation and Treatment of Patients with Cleft Lip/ Palate or Other Craniofacial Anomalies. American Cleft Palate-Craniofacial Association. Cleft Palate-Craniofacial Journal 2009; 30 (Suppl 1).

Peterson-Falzone, S. J., Trost-Cardamone, J. E., Karnell, M. P., \& Hardin-Jones, M. A. (2017). The clinicians guide to treating cleft palate speech. St. Louis, MO: Elsevier.

Pinheiro J, Bates D, DebRoy S, Sarkar D and R Core Team (2016). nlme: Linear and Nonlinear Mixed Effects Models. R package version 3.1-128, <URL: http://CRAN.R-project.org/package=nlme>.

R Core Team (2016). R: A Language and Environment for Statistical Computing. R Foundation for Statistical Computing, Vienna, Austria. <URL: https://www.R-project.org/>.

Revelle W (2016). psych: Procedures for Psychological, Psychometric, and Per- sonality Research. Northwestern University, Evanston, Illinois. R package version 1.6.9, <URL: https://CRAN.R-project.org/package=psych>.

Scherer, N., Kaiser, A., Frey, J., Roberts, M. Y., \& Lancaster, H. (2017). Effects of a naturalistic intervention on the speech outcomes of young children with cleft palate. Manuscript submitted for publication.

Sosa, Anna V., \& Stoel-Gammon, Carol. (2012). Lexical and Phonological Effects in Early Word Production. Journal of Speech, Language, and Hearing Research, 55(2), 596-608.

Stoel-Gammon, Carol. (2011). Relationships between Lexical and Phonological Development in Young Children. Journal of Child Language, 38(1), 1-34.

Stoel-Gammon, Carol, \& Cooper, Judith A. (1984). Patterns of Early Lexical and Phonological Development. Journal of Child Language, 11(2), 247-71.

Stoel-Gammon, C., \& Williams, A. (2013). Early phonological development: Creating an assessment test. Clinical Linguistics \& Phonetics, 2013, Vol.27(4), P.278-286, 27(4), 278-286.

Wickham H (2007). Reshaping Data with the reshape Package. Journal of Statistical Software, 21(12), pp. 1-20. <URL: http://www.jstatsoft.org/v21/i12/>.

Wickham H (2009). ggplot2: Elegant Graphics for Data Analysis. Springer-Verlag New York. ISBN 978-0-387-98140-6, <URL: http://ggplot2.org>.

Wickham H (2016). readxl: Read Excel Files. R package version 0.1.1, <URL: https://CRAN.R-project.org/package=readxl>.

Wickham H (2017). tidyr: Easily Tidy Data with 'spread()' and 'gather()' Functions. R package version 0.6.1, <URL: https://CRAN.R-project.org/package $=$ tidyr $>$.

Wickham H and Francois R (2016). dplyr: A Grammar of Data Manipulation. R package version 0.5.0, <URL: https://CRAN.R-project.org/package=dplyr $>$.

Vovakes, A., Philp, J., Lancaster, H., Lien, K., \& Scherer, S. (2017). Vocabulary predictors of early intervention outcomes for children with $\mathrm{CP}+/$ - L. Poster presented at Arizona State Univerity Department of Speech and Hearing Science Research Day, Tempe, AZ.

Xie Y (2016). knitr: A General-Purpose Package for Dynamic Report Generation in R. R package version 1.15, <URL: http://yihui.name/knitr/>. 